\title{
Potencial eólico na direção predominante do vento no Nordeste brasileiro
}

\author{
Bernardo B. da Silva ${ }^{1}$, Jakson J. A. Alves ${ }^{2}$, Enilson P. Cavalcanti ${ }^{3}$ \& Renilson T. Dantas ${ }^{4}$ \\ 1 DCA/CCT/UFCG. Av. Aprígio Veloso 882, Bloco CL, CEP 58109-970, Campina Grande, PB. E-mail: bernardo@dca.ufpb.br (Foto) \\ 2 UFCG. E-mail: jakson@dca.ufpb.br \\ ${ }^{3}$ DCA/CCT/UFCG. E-mail: enilson@dca.ufpb.br \\ ${ }^{4}$ DCA/CCT/UFCG. E-mail: renilson@dca.ufpb.br
}

Protocolo $15-22 / 2 / 2002$

\begin{abstract}
Resumo: Neste trabalho, foram utilizados dados horários de velocidade e direção do vento, provenientes de registros de anemógrafo Universal Fuess, a $10 \mathrm{~m}$ de altura, de 77 estações climatológicas pertencentes ao Instituto Nacional de Meteorologia (INMET), no período de janeiro de 1977 a dezembro de 1981. O objetivo da pesquisa foi a determinação da potência eólica média horária da direção predominante do vento em todas as estações selecionadas. Para tanto, identificou-se a direção predominante do vento de cada localidade e se estimaram os parâmetros da distribuição de Weibull, através dos quais se calculou a potência eólica média horária. Em função dos resultados apresentados neste trabalho, constatou-se que a direção predominante do vento no Nordeste do Brasil é de Leste, com flutuações para Sudeste e Nordeste. O ajuste do Método dos Momentos evidenciou-se melhor que o dos Mínimos Quadrados. Os Estados do Maranhão e Rio Grande do Norte apresentaram, respectivamente, o menor e maior potencial eólico. As estações de Alto Parnaíba, MA, e Acaraú, CE, apresentaram, mensalmente, a menor $\left(0,022 \mathrm{~W} \mathrm{~m}^{-2}\right)$ e a maior $\left(138,302 \mathrm{~W} \mathrm{~m}^{-2}\right)$ potência eólica, respectivamente.
\end{abstract}

Palavras-chave: distribuição de Weibull, potência eólica, climatologia do Nordeste

\section{Wind energy potential for the prevailing direction in Northeast Brazil}

\begin{abstract}
Hourly mean data of wind speed and direction, measured with a Fuess anemograph at $10 \mathrm{~m}$ height, were used in this study. These data were collected from January 1977 to December 1981 at 77 weather stations located in the Northeast of Brazil. The main objective of the study was to determine the wind energy potential for the predominant wind direction of selected stations; therefore, the identification of the relative frequency related to the hourly mean wind speed of the predominant direction was carried out. Then, the parameters, of a Weibull distribution related to the hourly mean wind speed of the predominant direction at each station were estimated by Moments and Graphical methods. The results show that the predominant wind direction in Northeast as a whole is East Ward, with variation to Northeast and Southeast. The Weibull model fitted very well to the observed frequency distribution of the wind speed data used in this research. The lowest and the highest wind energy potential were observed in the States of Maranhão and Rio Grande do Norte, respectively. At Acaraú (Ceará) the highest monthly wind energy potential, of $138.30 \mathrm{~W} \mathrm{~m}^{-2}$ was observed. On the other hand, the lowest monthly wind energy potential $\left(0.022 \mathrm{~W} \mathrm{~W} \mathrm{~m}^{-2}\right)$ was registered at Alto Parnaíba, MA.
\end{abstract}

Key words: Weibull distribution, wind power, climatology of Northeast Brazil

\section{INTRODUÇÃO}

Um dos grandes problemas enfrentados pela sociedade contemporânea diz respeito à questão energética, em virtude do seu consumo ser cada vez maior e de, ainda, prevalecer grande dependência dos recursos não renováveis, que têm no petróleo a sua principal fonte de matéria-prima. Ao persistir a atual taxa de consumo, as reservas mundiais poderão se esgotar dentro de algumas décadas. Algumas nações, antevendo as graves conseqüências dessa dependência, têm dedicado alguma atenção aos projetos relacionados com a utilização de fontes de energia alternativas. Dentre essas fontes, uma tem recebido grande destaque: a energia eólica, notadamente por ser renovável, "limpa" e de baixo custo, quando comparada com outras fontes de energia renováveis, como a solar, por exemplo. Quanto à relevância do seu caráter não poluente, 
considere-se que a geração de 1.649 GW h de energia extraída dos ventos, em substituição a outras fontes convencionais,

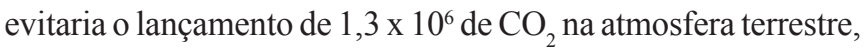
o que representaria contribuição de grande importância para o ambiente global (Garcia et al., 1998).

A identificação do potencial eólico de uma localidade é tarefa fundamental e tem, como requisito básico e indispensável, a existência de uma série temporal de observações da velocidade e direção do vento a uma altura adequada. No Nordeste brasileiro há registros de velocidades médias horárias do vento, obtidas a $10 \mathrm{~m}$ de altura, em 77 estações climatológicas pertencentes ao Instituto Nacional de Meteorologia (INMET). Com base em tais registros, Bastos et al. (1986) realizaram um zoneamento eólico do Nordeste brasileiro e obtiveram informações importantes sobre a potência média diária, ao longo do ano. Nesse estudo, não se utilizou nenhum tipo de distribuição probabilística nem foram identificadas as direções predominantes do vento. Anos mais tarde, Bandeira (1990) agrupou as velocidades médias horárias do vento em quatro períodos diários de seis horas cada um, e ajustou o modelo de Weibull às referidas médias e, mais uma vez, não foi identificada a potência eólica da direção predominante do vento.

Estudos sobre a exploração do vento como potencial energético, têm mostrado que o seu aproveitamento depende do conhecimento pormenorizado de suas características sobre a área onde serão implantadas as fazendas eólicas. Como o Nordeste brasileiro é influenciado pelos ventos alísios e eles oscilam de intensidade e direção entre o oceano e o continente, tais estudos devem caracterizar a variabilidade do potencial existente entre a zona costeira e a zona continental. Para produzir este conhecimento, tem sido amplamente usado o modelo de Weibull de dois parâmetros, em virtude do mesmo apresentar um bom ajuste à distribuição de freqüência dos dados de vento. Esses parâmetros têm sido classicamente estimados segundo o Método Gráfico, mais recentemente classificado como dos Mínimos Quadrados. Nos dias atuais estão sendo mais utilizados os métodos da Máxima Verossimilhança e o dos Momentos, pois são os que oferecem estimativas mais precisas dos parâmetros de Weibull.

Sopian et al. (1995) identificaram o potencial eólico de 10 estações da Malásia. O potencial máximo foi registrado em Mersing, com 85,61 $\mathrm{W} \mathrm{m}^{-2}$ a $10 \mathrm{~m}$ de altura, mas esses autores não obtiveram o potencial eólico da direção predominante do vento. Tolun et al. (1995) avaliaram o potencial eólico do Noroeste da Turquia e fizeram uso de vários modelos estatísticos, inclusive o de Weibull, e algoritmo que eliminava efeitos da orografia, rugosidade e obstáculos da superfície. O potencial foi avaliado em três alturas: 10, 30 e 50 m. Na estação NWS, a potência variou de 34 a $146 \mathrm{~W} \mathrm{~m}^{-2}$, para altura de $10 \mathrm{~m}$, e de 80 a $326 \mathrm{~W} \mathrm{~m}^{-2}$, a $50 \mathrm{~m}$.

O potencial eólico de Campo Grande e Dourado, MT, foi investigado por Sousa \& Granja (1997) a partir do registro de seis anos de dados do INMET, ocasião em que obtiveram os parâmetros de Weibull, para períodos semanais e mensais, baseados na velocidade e direção do vento medidos a $10 \mathrm{~m}$. Concluíram que as densidades de potência média anual em Campo Grande e Dourado foram, respectivamente, de 40,2 e 49,8 $\mathrm{W} \mathrm{m}^{-2}$ e que o Método Gráfico foi o que proporcionou a melhor estimativa dos parâmetros de Weibull, pois resultou nos menores erros dentre os métodos apresentados por Justus et al. (1976).

Mayhoub \& Azzam (1997) estimaram o potencial eólico de 15 estações no Egito, aos níveis de 10 e 25 m de altura, segundo os parâmetros de Weibull, que foram obtidos através do método gráfico. Na costa do Mar Vermelho, a potência chegou a superar os $450 \mathrm{~W} \mathrm{~m}^{-2}$. Em algumas estações do Mediterrâneo a energia eólica foi da ordem de 150 a $200 \mathrm{~kW} \mathrm{~h} \mathrm{~m}^{-2} \mathrm{ano}^{-1}$, enquanto no interior daquele país os valores caíram abaixo de $50 \mathrm{~kW} \mathrm{~h} \mathrm{~m}{ }^{-2}$ ano $^{-1}$. Na Arábia Saudita, Sahin \& Aksakal (1998) usaram as distribuições de Weibull e Rayleight para avaliar o desempenho de um sistema eólico e concluíram que os resultados alcançados com os dados experimentais se aproximaram melhor daqueles gerados com o modelo de Weibull, com erro relativo de apenas $3,46 \%$.

Os mecanismos atmosféricos responsáveis pelo alto potencial eólico e ilhas vizinhas foram investigados por Borhan (1998), que quantificou a potência eólica de três localidades com a distribuição de Weibull e verificou que em Bozcoada a potência a $30 \mathrm{~m}$ situou-se entre 120,5 a $1.410,9 \mathrm{~W} \mathrm{~m}^{-2}$. As características do vento e o potencial eólico do Marrocos (Nfaoui et al., 1998), da Turquia (Borhan, 1998) e da Argélia (Merzouk, 2000) foram igualmente obtidos através da distribuição de Weibull e registros eólicos com diferentes durações.

Não obstante a existência de algumas pesquisas sobre o potencial eólico do Nordeste do Brasil, constata-se que não há estudo acerca da identificação de tal potencial para uma direção particular do vento, mesmo sendo esta uma informação imprescindível à instalação de turbinas eólicas de grande porte. Com o exposto, a presente pesquisa objetiva a determinação da potência eólica da direção predominante do vento das 77 Estações Climatológicas do Nordeste do Brasil.

\section{MATERIAL E MÉTODOS}

Os dados que serviram de base para o presente estudo consistem de velocidade e direção horária do vento, registrados em anemógrafos Fuess, instalados em 77 localidades da rede de estações climatológicas do Instituto Nacional de Meteorologia (INMET). As estações climatológicas utilizadas na pesquisa são apresentadas na Tabela 1 , com os seus respectivos códigos e coordenadas de posição. Foram consideradas todas as medições obtidas no período de janeiro de 1977 a dezembro de 1981, inclusive. O elemento sensível dos anemógrafos situavase a $10 \mathrm{~m}$ da superfície e proporcionou a obtenção da direção horária predominante do vento e a velocidade média horária correspondente. Foram consideradas, para fins de identificação da direção horária predominante, oito faixas centradas nas direções que se encontram na Tabela 2. Cada faixa corresponde a $45^{\circ}$, e a direção Norte, por exemplo, compreende os ventos com direções entre 337,5 a $360^{\circ}$ e de 0 a $22,5^{\circ}$.

Um programa em FORTRAN 90 foi desenvolvido para gerar a distribuição de freqüências associada a cada uma das direções 
Tabela 1. Relação das estações climatológicas incluídas na pesquisa, com seus respectivos códigos e coordenadas de posição

\begin{tabular}{|c|c|c|c|c|c|c|c|}
\hline Código & Estação & Lat (S) & Long (W) & Código & Estação & Lat (S) & Long (W) \\
\hline \multicolumn{4}{|c|}{ Rio Grande do Norte } & Pernambuco & & & \\
\hline 82590 & Apodi & $5^{\circ} 39^{\prime}$ & $37^{\circ} 48^{\prime}$ & 82753 & Ouricuri & $7^{\circ} 54^{\prime}$ & $40^{\circ} 03^{\prime}$ \\
\hline 82594 & Macau & $5^{\circ} 06^{\prime}$ & $36^{\circ} 37^{\prime}$ & 82789 & Triunfo & $7^{\circ} 51^{\prime}$ & $38^{\circ} 08^{\prime}$ \\
\hline 82599 & Natal & $5^{\circ} 39^{\prime}$ & $35^{\circ} 00^{\prime}$ & 82797 & Surubim & $7^{\circ} 50^{\prime}$ & $35^{\circ} 46^{\prime}$ \\
\hline 82598 & Ceará Mirim & $5^{\circ} 39^{\prime}$ & $35^{\circ} 25^{\prime}$ & 82879 & Arcoverde & $8^{\circ} 20^{\prime}$ & $36^{\circ} 45^{\prime}$ \\
\hline 82696 & Cruzeta & $6^{\circ} 26^{\prime}$ & $36^{\circ} 47^{\prime}$ & 82886 & Cabrobó & $8^{\circ} 31^{\prime}$ & $39^{\circ} 18^{\prime}$ \\
\hline Paraíba & & & & 82893 & Garanhuns & $8^{\circ} 53^{\prime}$ & $36^{\circ} 29^{\prime}$ \\
\hline Paraiba & & & & 82900 & Recife & $8^{\circ} 03^{\prime}$ & $34^{\circ} 55^{\prime}$ \\
\hline 82689 & São Gonçalo & $6^{\circ} 45^{\prime}$ & $38^{\circ} 13^{\prime}$ & 82983 & Petrolina & $9^{\circ} 23^{\prime}$ & $40^{\circ} 30^{\prime}$ \\
\hline 82791 & Patos & $7^{\circ} 00^{\prime}$ & $37^{\circ} 16^{\prime}$ & \multirow{2}{*}{\multicolumn{4}{|c|}{ Maranhão }} \\
\hline 82792 & Monteiro & $7^{\circ} 53^{\prime}$ & $37^{\circ} 07^{\prime}$ & & & & \\
\hline 82795 & Campina Grande & $7^{\circ} 13^{\prime}$ & $35^{\circ} 53^{\prime}$ & 82198 & Turiaçu & $1^{\circ} 43^{\prime}$ & $45^{\circ} 24^{\prime}$ \\
\hline 82798 & João Pessoa & $7^{\circ} 07^{\prime}$ & $34^{\circ} 53^{\prime}$ & 82280 & São Luiz & $2^{\circ} 32^{\prime}$ & $44^{\circ} 17^{\prime}$ \\
\hline & & & & 82376 & Zé Doca & $3^{\circ} 42^{\prime}$ & $45^{\circ} 34^{\prime}$ \\
\hline P1auı & & & & 82382 & Chapadinha & $3^{\circ} 44^{\prime}$ & $43^{\circ} 25^{\prime}$ \\
\hline 82288 & Parnaíba & $2^{\circ} 55^{\prime}$ & $41^{\circ} 36^{\prime}$ & 82460 & Bacabal & $4^{\circ} 45^{\prime}$ & $44^{\circ} 45^{\prime}$ \\
\hline 82480 & Piripiri & $4^{\circ} 16^{\prime}$ & $41^{\circ} 47^{\prime}$ & 82476 & Caxias & $4^{\circ} 54^{\prime}$ & $43^{\circ} 24^{\prime}$ \\
\hline 82578 & Teresina & $5^{\circ} 05^{\prime}$ & $42^{\circ} 49^{\prime}$ & 82564 & Imperatriz & $5^{\circ} 34^{\prime}$ & $47^{\circ} 35^{\prime}$ \\
\hline 82669 & São Félix do Piauí & $5^{\circ} 58^{\prime}$ & $42^{\circ} 10^{\prime}$ & 82571 & Barra do Corda & $5^{\circ} 30^{\prime}$ & $45^{\circ} 16^{\prime}$ \\
\hline 82678 & Floriano & $6^{\circ} 07^{\prime}$ & $42^{\circ} 50^{\prime}$ & 82676 & Colinas & $6^{\circ} 03$ & $44^{\circ} 18^{\prime}$ \\
\hline 82771 & Uruçuí & $7^{\circ} 10^{\prime}$ & $44^{\circ} 22^{\prime}$ & 82765 & Carolina & $7^{\circ} 20^{\prime}$ & $47^{\circ} 18^{\prime}$ \\
\hline 82780 & Picos & $7^{\circ} 05^{\prime}$ & $41^{\circ} 27^{\prime}$ & 82970 & Alto Parnaíba & $9^{\circ} 07^{\prime}$ & $45^{\circ} 56^{\prime}$ \\
\hline 82879 & São João do Piauí & $8^{\circ} 21^{\prime}$ & $42^{\circ} 51^{\prime}$ & \multirow{2}{*}{\multicolumn{4}{|c|}{ Bahia }} \\
\hline 82882 & Paulistana & $8^{\circ} 08^{\prime}$ & $41^{\circ} 08^{\prime}$ & & & & \\
\hline 82975 & Bom Jesus do Piauí & $9^{\circ} 06^{\prime}$ & $44^{\circ} 07^{\prime}$ & 82979 & Remanso & $9^{\circ} 41^{\prime}$ & $42^{\circ} 44^{\prime}$ \\
\hline 82976 & Caracol & $9^{\circ} 10^{\prime}$ & $43^{\circ} 20^{\prime}$ & 82986 & Paulo Afonso & $9^{\circ} 21$ & $38^{\circ} 51^{\prime}$ \\
\hline Ceará & & & & 83076 & Ibipetuba & $11^{\circ} 01^{\prime}$ & $44^{\circ} 31^{\prime}$ \\
\hline Ceara & & & & 83090 & Monte Santo & $10^{\circ} 26^{\prime}$ & $39^{\circ} 02^{\prime}$ \\
\hline 82294 & Acaraú & $2^{\circ} 53^{\prime}$ & $40^{\circ} 08^{\prime}$ & 83179 & Barra & $11^{\circ} 05^{\prime}$ & $43^{\circ} 12^{\prime}$ \\
\hline 82392 & Sobral & $3^{\circ} 42^{\prime}$ & $40^{\circ} 21^{\prime}$ & 83182 & Irecê & $11^{\mathrm{o}} 18^{\prime}$ & $41^{\circ} 54^{\prime}$ \\
\hline 82397 & Fortaleza & $3^{\circ} 51^{\prime}$ & $38^{\circ} 36^{\prime}$ & 83186 & Jacobina & $11^{\circ} 11^{\prime}$ & $40^{\circ} 31^{\prime}$ \\
\hline 82487 & Guaramiranga & $4^{\circ} 17^{\prime}$ & $39^{\circ} 09^{\prime}$ & 83190 & Serrinha & $11^{\mathrm{o}} 38^{\prime}$ & $38^{\circ} 59^{\prime}$ \\
\hline 82493 & Jaguaruana & $4^{\circ} 50^{\prime}$ & $37^{\circ} 48^{\prime}$ & 83229 & Salvador & $13^{\circ} 00^{\prime}$ & $38^{\circ} 31^{\prime}$ \\
\hline 82583 & Crateús & $5^{\circ} 10^{\prime}$ & $40^{\circ} 44^{\prime}$ & 83236 & Barreiras & $12^{\circ} 08^{\prime}$ & $45^{\circ} 00^{\prime}$ \\
\hline 82586 & Quixeramobim & $5^{\circ} 12^{\prime}$ & $39^{\circ} 18^{\prime}$ & 83242 & Lençóis & $12^{\circ} 34^{\prime}$ & $41^{\circ} 23^{\prime}$ \\
\hline 92588 & Morada Nova & $5^{\circ} 06^{\prime}$ & $38^{\circ} 23^{\prime}$ & 83244 & Itaberaba & $12^{\circ} 33^{\prime}$ & $40^{\circ} 17^{\prime}$ \\
\hline 82686 & Iguatu & $6^{\circ} 22^{\prime}$ & $39^{\circ} 18^{\prime}$ & 83249 & Alagoinhas & $12^{\circ} 08^{\prime}$ & $38^{\circ} 25^{\prime}$ \\
\hline 82784 & Barbalha & $7^{\circ} 19^{\prime}$ & $39^{\circ} 18^{\prime}$ & 83286 & Correntina & $13^{\circ} 20^{\prime}$ & $44^{\circ} 36^{\prime}$ \\
\hline Sergipe & & & & 83288 & Bom Jesus da Lapa & $13^{\circ} 16^{\prime}$ & $43^{\circ} 52^{\prime}$ \\
\hline 83096 & Aracaju & $10^{\circ} 54^{\prime}$ & $37^{\circ} 04^{\prime}$ & 83295 & Jaguaquara & $13^{\circ} 32^{\prime}$ & $39^{\circ} 57^{\prime}$ \\
\hline 83097 & Propriá & $10^{\circ} 13^{\prime}$ & $36^{\circ} 51^{\prime}$ & 83339 & Caetité & $14^{\circ} 04^{\prime}$ & $42^{\circ} 28^{\prime}$ \\
\hline Alagoas & & & & 83344 & Vitoria da Conquista & $14^{\circ} 57^{\prime}$ & $40^{\circ} 53^{\prime}$ \\
\hline & & & & 83348 & Ilhéus & $14^{\circ} 47^{\prime}$ & $39^{\circ} 03^{\prime}$ \\
\hline 82992 & Palmeira dos Índios & $9^{\circ} 25^{\prime}$ & $36^{\circ} 40^{\prime}$ & 83408 & Carinhanha & $14^{\circ} 10^{\prime}$ & $43^{\circ} 55^{\prime}$ \\
\hline 82994 & Maceió & $9^{\circ} 40^{\prime}$ & $35^{\circ} 43^{\prime}$ & 83446 & Guaratinga & $16^{\circ} 25^{\prime}$ & $39^{\circ} 35^{\prime}$ \\
\hline 83098 & Coruripe & $10^{\circ} 07^{\prime}$ & $36^{\circ} 11^{\prime}$ & 83498 & Caravelas & $17^{\circ} 44^{\prime}$ & $39^{\circ} 15^{\prime}$ \\
\hline
\end{tabular}

Tabela 2. Codificação usada na classificação da direção horária predominante

\begin{tabular}{clc}
\hline Identificação & Denominação & Direção \\
\hline 1 & Norte & $360^{\circ}$ \\
2 & Nordeste & $45^{\circ}$ \\
3 & Leste & $90^{\circ}$ \\
4 & Sudeste & $135^{\circ}$ \\
5 & Sul & $180^{\circ}$ \\
6 & Sudoeste & $225^{\circ}$ \\
7 & Oeste & $270^{\circ}$ \\
8 & Noroeste & $315^{\circ}$ \\
\hline
\end{tabular}

consideradas na Tabela 2. Uma vez identificada a direção predominante de cada estação climatológica, passou-se à fase de análise da distribuição de freqüências das velocidades correspondentes apenas àquela direção predominante. Nessa etapa, foram determinadas as freqüências acumuladas segundo a freqüência Kimbal e os parâmetros da distribuição de Weibull, cujos procedimentos são descritos a seguir.

\section{O modelo de Weibull}

A distribuição de Weibull tem sido utilizada em inúmeros estudos destinados à análise de freqüência da velocidade do vento, e sua função de densidade de probabilidade é do tipo:

$$
f(x)=\left(\frac{a}{b}\right)\left(\frac{x}{b}\right)^{a-1} \exp \left[-\left(\frac{x}{b}\right)^{a}\right]
$$

em que:

a - parâmetro de forma (adimensional e positivo) 
b - fator de escala ( $\mathrm{m} \mathrm{s}^{-1}$ e positivo)

x - velocidade do vento $\left(\mathrm{m} \mathrm{s}^{-1}\right)$

A função de distribuição $\mathrm{F}(\mathrm{x})$, associada ao modelo probabilístico de Weibull, é dada por:

$$
F(x)=1-\exp \left[-\left(\frac{x}{b}\right)^{a}\right]
$$

Os parâmetros do modelo Weibull foram estimados segundo os métodos dos Mínimos Quadrados (ou Método Gráfico) e dos Momentos. O Método Gráfico é o que tem sido mais amplamente utilizado, principalmente antes da utilização massiva de microcomputadores.

No Método Gráfico determinam-se a e b, através da solução da seguinte equação:

$$
\ln \{-\ln [1-\mathrm{F}(\mathrm{x})]\}=-\mathrm{a} \ln (\mathrm{b})+\mathrm{a} \ln (\mathrm{x})
$$

em que $\mathrm{F}(\mathrm{x})$ é substituída pela freqüência empírica de Kimball (Silva et al., 2001). O procedimento de cálculo dos valores de a e b, a partir dessa etapa, consiste em se considerar uma equação linear do tipo: $\mathrm{Y}=\mathrm{a}_{\mathrm{o}}+\mathrm{b}_{\mathrm{o}} \mathrm{X}$, em que $\mathrm{a}_{\mathrm{o}}=-\mathrm{a} \ln (\mathrm{b}), \mathrm{b}_{\mathrm{o}}=\mathrm{a}$ e $\mathrm{X}=\ln (\mathrm{x})$.

No Método dos Momentos são obtidos os momentos de primeira $[\mathrm{E}(\mathrm{x})]$ e segunda $\left[\mathrm{E}\left(\mathrm{x}^{2}\right)\right]$ ordens, centrados na origem, que para o modelo de Weibull, são dados, respectivamente, por:

$$
\mathrm{E}(\mathrm{x})=\mathrm{b} \Gamma(1+1 / \mathrm{a})
$$

e

$$
\mathrm{E}\left(\mathrm{x}^{2}\right)=\mathrm{b}^{2} \Gamma(1+2 / \mathrm{a})
$$

A variância de $\mathrm{x}, \operatorname{Var}(\mathrm{x})$, por definição, é dada por $\mathrm{E}\left(\mathrm{x}^{2}\right)$ $[\mathrm{E}(\mathrm{x})]^{2}$ o que resulta, para a distribuição de Weibull, na seguinte igualdade:

$$
\operatorname{Var}(\mathrm{x})=\mathrm{b}^{2}\left[\Gamma(1+2 / \mathrm{a})-\Gamma^{2}(1+1 / \mathrm{a})\right]
$$

A solução da função matemática gama $(\Gamma)$ pode ser aproximada por polinômio (Abramowitz \& Stegun, 1972). Substituindo-se na Eq. (6) o valor de b obtido na Eq. (4), e considerando-se que a média aritmética amostral $(\mathrm{xm})$ e o desvio-padrão ao quadrado $\left(\mathrm{s}^{2}\right)$ são estimadores da esperança matemática e variância da distribuição de Weibull, respectivamente, tem-se:

$$
\mathrm{s}^{2}=\mathrm{xm}^{2}\left(\frac{\Gamma(1+2 / \mathrm{a})}{\Gamma^{2}(1+1 / \mathrm{a})}-1\right)
$$

A determinação de a é obtida com base na solução da equação:

$$
f(a)=s^{2}-x m^{2}\left(\frac{\Gamma(1+2 / a)}{\Gamma^{2}(1+1 / a)}-1\right)
$$

que pode ser resolvida por método numérico destinado à obtenção da raiz de uma equação.

A densidade de potência eólica $(\mathrm{P})$ média horária $\left(\mathrm{W} \mathrm{m}^{-2}\right)$ foi calculada com base nos parâmetros da distribuição de Weibull, obtidos pelos dois métodos já mencionados e na freqüência relativa correspondente à direção predominante do vento, em cada uma das 77 estações climatológicas incluídas no estudo, segundo a expressão:

$$
P=\frac{1}{2} \rho E\left(x^{3}\right) f r(d)
$$

em que:

$\rho \quad$ - massa específica do ar úmido $\left(1,15 \mathrm{~kg} \mathrm{~m}^{-3}\right)$

fr(d) - freqüência relativa correspondente à direção predominante do vento

$\mathrm{E}\left(\mathrm{x}^{3}\right)$ - terceiro momento centrado na origem que, para a distribuição de Weibull, é igual a :

$$
\mathrm{E}\left(\mathrm{x}^{3}\right)=\mathrm{b}^{3} \Gamma(1+3 / \mathrm{a})
$$

em que:

a e b - parâmetros de Weibull

$\Gamma \quad$ - função matemática gama

\section{RESULTADOS E DISCUSSÃO}

\section{Análise da direção predominante}

Os dados correspondentes à direção predominante (DP) do vento, de cada uma das 77 estações selecionadas para o estudo, são apresentados na Tabela 3. Para tanto, foram consideradas todas as medições da velocidade média horária e direção correspondente. Após a identificação da direção predominante de cada mês, procedeu-se à identificação da DP anual, ou seja, daquela que ocorre com maior freqüência ao longo do ano. Nos casos em que ocorreram empates no número de meses associados às direções predominantes, escolheu-se, como DP anual, aquela detentora da maior velocidade média.

No Estado de Sergipe foram consideradas as localidades de Propriá e Aracaju, esta com direção predominante 4, embora em quatro meses do ano tenha ocorrido a direção 3, ou seja, Leste. Em Propriá, a DP foi Leste, verificada em nove meses do ano, e a outra DP mensal foi a Sudeste; já em Alagoas a DP mensal dos ventos apresentou comportamento semelhante ao verificado em Sergipe, exceto que foi registrada DP de Nordeste em Coruripe nos meses janeiro, novembro e dezembro. Em Maceió predominou a direção Sudeste, enquanto em Palmeira dos Índios e Coruripe predominou a direção 3, Leste. 
Tabela 3. Direção predominante mensal dos ventos nas localidades estudadas e direção predominante anual (DP)

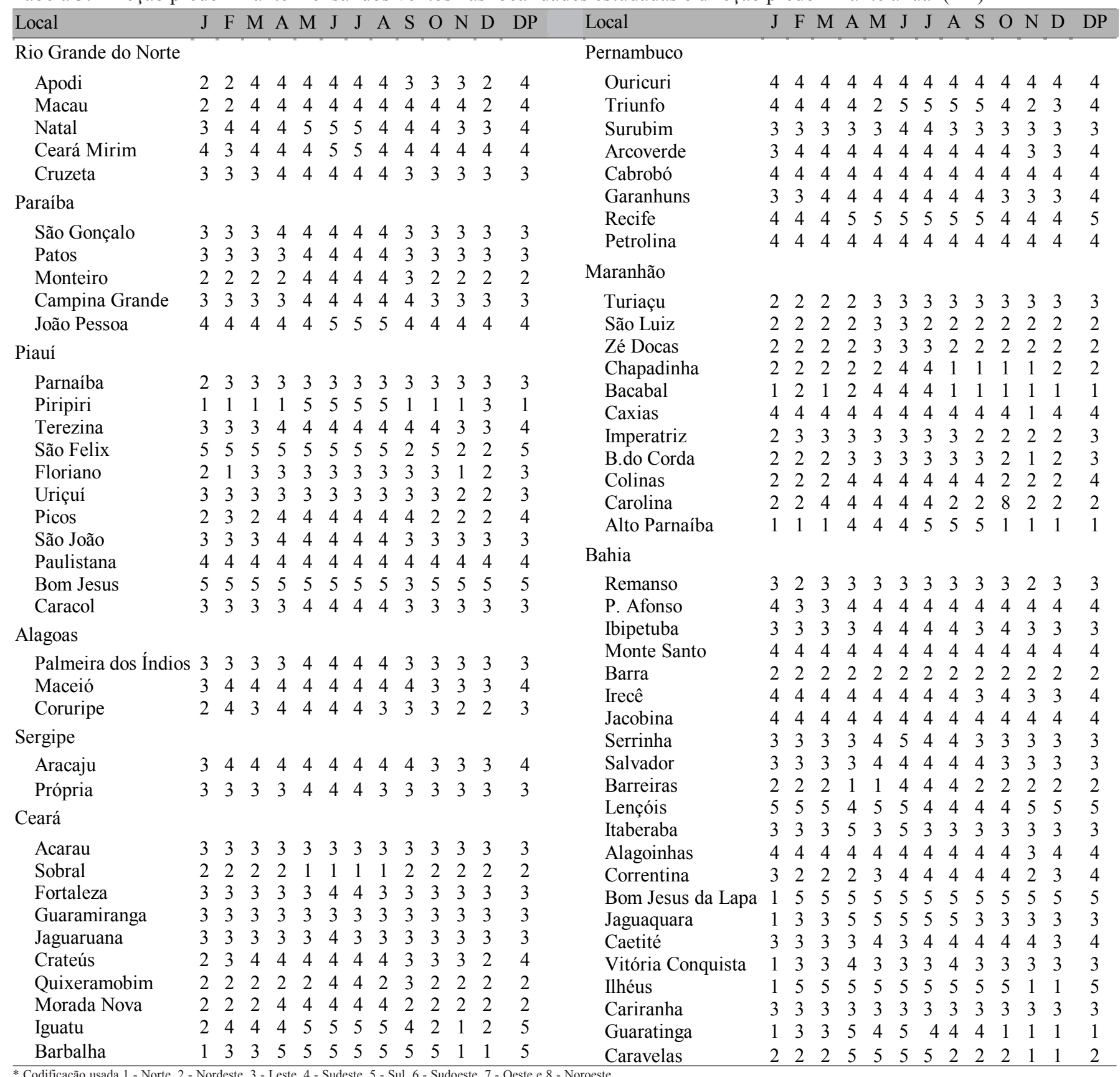

* Codificação usada 1 - Norte, 2 - Nordeste, 3 - Leste, 4 - Sudeste, 5 - Sul, 6 - Sudoeste, 7 - Oeste e 8 - Noroeste

Nas estações do Rio Grande do Norte foram registradas DP mensais: 2, 3, 4 e 5, ou seja, Nordeste, Leste, Sudeste e Sul, respectivamente, embora com predominância 4 , com exceção de Cruzeta, que foi Leste, e nas demais localidades predominou a direção Sudeste. Por outro lado, no Estado da Paraíba foram registradas DP mensais correspondentes aos quadrantes Nordeste, Leste e Sudeste. A estação de Monteiro apresentou direção muito variável, mas com predominância de Nordeste em sete meses do ano. Em Campina Grande, Patos e São Gonçalo, predominou direção Leste, enquanto João Pessoa teve, como predominante, as direções Sudeste.

No Estado de Pernambuco o comportamento da direção predominante é semelhante ao verificado nos Estados da Paraíba e Rio Grande do Norte, ou seja, as direções Leste, Sudeste e Sul, com predominância no Estado como um todo, da direção Sudeste. Fato marcante foi observado em Petrolina,
Ouricuri e Cabrobó, onde se registrou apenas a DP 4, Sudeste, em todo o ano. Com relação à direção predominante de cada localidade, observou-se que Arcoverde, Cabrobó, Garanhuns, Ouricuri e Petrolina apresentaram a DP anual Sudeste, enquanto Recife e Triunfo apresentaram a DP Sul, e apenas Surubim apresentou a DP anual Leste.

No Ceará, a DP mensal mostrou-se muito variável, indo de Norte a Sul. Fortaleza, Acaraú, Jaguaruana e Guarani, apresentaram a direção 3 (Leste), enquanto Sobral, Quixeramobim e Morada Nova, apresentaram DP Nordeste; Barbalha e Iguatu, Sul e apenas Crateús apresentou DP Sudeste. No Estado, a DP foi a Leste. As localidades Barbalha, Sobral e Iguatu foram as únicas a registrar direção Norte. No Maranhão, também foi observada grande variabilidade na DP, e foram registradas as direções: Noroeste (mês de outubro em Carolina), Norte, Nordeste, Leste, Sudeste e Sul. Em todas as estações 
foram registradas pelo menos duas DP mensais diferentes. São Luiz, Chapadinha, Carolina e Zé Docas, apresentaram DP Nordeste; já em Imperatriz, Turiaçu e Barra do Corda, a DP foi Leste; Alto Parnaíba e Bacabal apresentaram DP Norte, enquanto Colinas e Caxias registraram DP Sudeste.

No Estado do Piauí foram observadas as seguintes DPs: Norte, Nordeste, Leste, Sudeste e Sul. As únicas localidades a registrarem direção Norte, foram Piripiri (sete meses) e Floriano (dois meses apenas). Nesse Estado, como um todo, a DP foi Leste. A DP anual de Parnaíba, São João, Uriçuí e Caracol foi a Leste; já em Paulistana, Picos e Terezina, a DP anual foi a 4 (Sudeste). Em São Félix e Bom Jesus, predominou a direção Sul e, em Piripiri, a Norte.

O Estado da Bahia apresentou a maior variabilidade na direção predominante do vento, com ocorrência desde o Norte, passando por Nordeste, Leste, Sudeste e Sul, como pode ser observado na Tabela 3. Isto pode ser justificado pelas dimensões do Estado e pelo fato do mesmo estar submetido à ação de diferentes sistemas meteorológicos, tais como: sistemas frontais, alta semipermanente do Atlântico sul, ondas de leste, etc. As localidades de Carinhanha, Ibipetuba, Itaberaba, Jaguaquara, Remanso, Salvador e Serrinha, apresentaram a direção Leste; já Alagoinhas, Caetité Irecê, Jacobina, Monte Santo, Paulo Afonso e Correntina, apresentaram DP Sudeste; Barra, Barreiras e Caravelas, apresentaram a direção Nordeste; Bom Jesus da Lapa, Ilhéus e Lençóis, a direção Sul, e apenas Guaratinga apresentou a direção Norte. No Estado como um todo, a direção predominante foi a 3 (Leste).

O conhecimento da direção predominante permite avaliarse melhor o potencial eólico da região, seja em virtude das variações associadas à freqüência do vento, seja face às variações de direção, porém o rendimento esperado por uma máquina eólica fixada a partir da direção predominante escapa aos objetivos do presente trabalho. Para a região de Maringá, PR, Galvani et al. (1999) concluíram que a DP do vento anual nos horários das 12 e 18 GMT foi de Nordeste; no entanto, nos meses de janeiro a maio e de outubro a dezembro a DP às 24 GMT foi Sudeste, sendo Leste de julho a setembro. Souza \& Granja (1997) encontraram ventos predominantes de Leste em Campo Grande, MS, com ocorrência de Norte nos meses de janeiro a dezembro, cujos valores anuais resultaram em $24 \%$ de Leste, $19,8 \%$ de Norte e $12,2 \%$ de Nordeste, tendo as calmarias representado $12 \%$.

\section{Potência eólica}

Com base na identificação da direção predominante do vento de cada localidade pesquisada, procedeu-se à obtenção dos parâmetros a e b da distribuição de Weibull, segundo os métodos dos Momentos e dos Mínimos Quadrados (Gráfico); em seguida, identificou-se o método que proporcionou o melhor ajustamento aos dados da velocidade do vento da direção predominante. De modo geral, constatou-se que o Método dos Momentos proporcionou o melhor ajustamento, aferido pela diferença entre as distribuições empírica e de Weibull e, por esta razão, foi escolhido para o cálculo da densidade de potência instantânea média horária mensal (DPMM) e a sua média anual (DPMA) de todas as localidades incluídas na pesquisa.

$\mathrm{Na}$ Tabela 4 são apresentados os valores da DPMM de todos os meses e localidades estudadas. Como pode ser observado, dentre as localidades do Rio Grande do Norte,
Macau foi a que apresentou maior potência eólica associada à direção predominante, com média anual de 41,273 $\mathrm{W} \mathrm{m}^{-2}$. Ademais, a DPMM nesse mesmo local chegou a atingir, no mês de agosto, 92,329 $\mathrm{W} \mathrm{m}^{-2}$. No Estado da Paraíba, por sua vez, Campina Grande e São Gonçalo foram as localidades que apresentaram, respectivamente, o maior e o menor potencial eólico, com valores da DPMA iguais a 29,324 e 8,273 W m-2. O mês de novembro em Campina Grande foi o de maior potencial eólico, com 62,592 $\mathrm{W} \mathrm{m}^{-2}$. Destaque-se o potencial de João Pessoa, com DPMA de 21,621 $\mathrm{W} \mathrm{m}^{-2}$, inferior àquele verificado em Campina Grande e Patos. Este fato sugere uma avaliação mais pormenorizada de tais valores, que podem resultar de uma grande variabilidade da direção do vento que resulta, em potencial da direção predominante, não muito diferente daquele associado a outras direções do vento; ademais, outros fatores podem estar interferindo em tal potencial, como a existência de obstáculos nas proximidades do anemógrafo utilizado. Ao avaliarem o potencial eólico da Malásia, Sopian et al. (1995) observaram que o maior potencial foi obtido em Mersing, com DPMA igual a 85,615 W m² a $10 \mathrm{~m}$, alcançando $119,755 \mathrm{~W} \mathrm{~m}^{-2}$ no mês de janeiro; já Tolun et al. (1995) observaram que no noroeste da Turquia a DPMA chegou a $342 \mathrm{~W} \mathrm{~m}^{-2}$, observada na localidade de Cinaralti.

Em Pernambuco, a DPMA variou de 37,87 $\mathrm{W} \mathrm{m}^{-2}$, em Petrolina, a 12,14 $\mathrm{W} \mathrm{m}^{-2}$, em Recife. Isto ocorre, possivelmente, porque o litoral do Nordeste possui ventos persistentes ao longo dos dias e meses do ano, resultantes da ação do anticiclone semipermanente do Atlântico Sul e que são responsáveis pela ocorrência dos ventos alísios na região Nordeste. O desenvolvimento urbano em torno das estações poderia estar associado a tais discrepâncias. A nível mensal, a maior DPMM foi registrada no mês de setembro, em Petrolina.

Verificou-se que o maior e o menor potencial eólico do Estado de Alagoas ocorreram, respectivamente, em Palmeira dos Índios, com DPMA de 19,038 W m² ${ }^{-2}$ e Maceió, onde a DPMA foi igual a 7,090 $\mathrm{W} \mathrm{m}^{-2}$. Mais uma vez se constata que o potencial eólico da direção predominante no litoral foi menor que aquele obtido no interior do Estado; já em Sergipe, existem apenas duas localidades com registros anemométricos, sendo que o potencial eólico maior se localizou no litoral, mais precisamente em Aracaju, mas com uma DPMA de 24,757 $\mathrm{W} \mathrm{m}^{-2}$, menor ainda que o verificado em João Pessoa.

No Estado do Ceará, a DPMA variou de $64,321 \mathrm{Wm}^{-2}$, obtido em Acaraú, a 6,875 $\mathrm{W} \mathrm{m}^{-2}$, registrado em Iguatu. Em Fortaleza, a maior DPMM ocorreu no mês de outubro, com 37,820 W m², e apresentou DPMA igual a 19,614 W m². No Piauí, de maneira geral, foram obtidos potenciais muito baixos, exceto em Paulistana, que apresentou uma DPMA 55,251 W m², e Parnaíba, com DPMA 34,130 W m-2. Nas demais localidades desse Estado, a DPMA foi inferior aos $10,000 \mathrm{~W} \mathrm{~m}^{-2}$. Situação semelhante foi verificada no Estado do Maranhão, onde a maior potência eólica foi registrada em Turiaçu, com DPMA de 17,434 $\mathrm{W} \mathrm{m}^{-2}$. Nesta localidade, a DPMM alcançou o seu máximo valor em setembro, com DPMM 37,603 W m². Nas demais localidades desse Estado a DPMA foi inferior $10,00 \mathrm{~W} \mathrm{~m}^{-2}$; portanto, com potencial eólico muito baixo. Em Parnaíba, a DPMA foi de apenas $0,688 \mathrm{~W} \mathrm{~m}^{-2}$, sendo a menor dentre todas as localidades nordestinas estudadas. 
Tabela 4. Densidade de potência eólica instantânea média horária ( $\left.\mathrm{W} \mathrm{m}^{-2}\right)$ em todas as localidades selecionadas ao longo dos meses do ano

\begin{tabular}{|c|c|c|c|c|c|c|c|c|c|c|c|c|c|}
\hline \multirow{2}{*}{ Localidades } & \multicolumn{12}{|c|}{ Meses } & \multirow{2}{*}{ Média } \\
\hline & Jan & $\mathrm{Fev}$ & Mar & Abr & Mai & Jun & Jul & Ago & Set & Out & Nov & Dez & \\
\hline \multicolumn{14}{|l|}{ Rio Grande do Norte } \\
\hline $\begin{array}{l}\text { Apodi } \\
\text { Macau } \\
\text { Natal } \\
\text { Ceará Mirim } \\
\text { Cruzeta }\end{array}$ & $\begin{array}{r}1,7 \\
11,5 \\
22,6 \\
18,5 \\
21,9\end{array}$ & $\begin{array}{r}2,1 \\
10,8 \\
31,5 \\
21,5 \\
14,8\end{array}$ & $\begin{array}{r}2,8 \\
20,0 \\
24,1 \\
19,7 \\
8,9\end{array}$ & $\begin{array}{r}4,2 \\
33,3 \\
29,4 \\
25,2 \\
5,0\end{array}$ & $\begin{array}{r}5,2 \\
47,3 \\
36,1 \\
24,6 \\
4,1\end{array}$ & $\begin{array}{r}8,3 \\
51,6 \\
36,7 \\
22,7 \\
5,1\end{array}$ & $\begin{array}{r}11,1 \\
68,4 \\
48,0 \\
33,7 \\
6,2\end{array}$ & $\begin{array}{l}10,2 \\
92,3 \\
59,7 \\
39,0 \\
13,0\end{array}$ & $\begin{array}{r}8,3 \\
81,5 \\
64,6 \\
50,1 \\
28,9\end{array}$ & $\begin{array}{r}3,6 \\
47,2 \\
67,1 \\
47,9 \\
34,0\end{array}$ & $\begin{array}{r}1,4 \\
18,1 \\
33,2 \\
34,3 \\
36,8\end{array}$ & $\begin{array}{r}0,8 \\
13,2 \\
33,5 \\
30,3 \\
33,9\end{array}$ & $\begin{array}{r}5,0 \\
41,3 \\
40,5 \\
30,6 \\
17,7\end{array}$ \\
\hline \multicolumn{14}{|l|}{ Paraíba } \\
\hline $\begin{array}{l}\text { São Gonçalo } \\
\text { Patos } \\
\text { Monteiro } \\
\text { Campina Grande } \\
\text { João Pessoa }\end{array}$ & $\begin{array}{r}8,2 \\
25,8 \\
22,7 \\
40,9 \\
18,8\end{array}$ & $\begin{array}{r}5,5 \\
24,5 \\
14,7 \\
39,2 \\
19,5\end{array}$ & $\begin{array}{r}3,4 \\
11,7 \\
9,4 \\
26,2 \\
10,2\end{array}$ & $\begin{array}{r}2,2 \\
9,0 \\
5,1 \\
15,2 \\
15,8\end{array}$ & $\begin{array}{r}1,8 \\
11,0 \\
1,0 \\
8,9 \\
17,3\end{array}$ & $\begin{array}{r}2,7 \\
10,5 \\
1,3 \\
7,4 \\
20,0\end{array}$ & $\begin{array}{r}3,3 \\
14,4 \\
0,1 \\
7,0 \\
25,1\end{array}$ & $\begin{array}{r}6,9 \\
27,5 \\
1,2 \\
13,2 \\
21,8\end{array}$ & $\begin{array}{r}16,3 \\
43,7 \\
4,3 \\
28,3 \\
28,4\end{array}$ & $\begin{array}{l}19,2 \\
59,4 \\
23,3 \\
50,4 \\
32,1\end{array}$ & $\begin{array}{l}16,3 \\
45,3 \\
41,3 \\
62,6 \\
22,6\end{array}$ & $\begin{array}{l}13,5 \\
35,6 \\
31,4 \\
52,7 \\
27,8\end{array}$ & $\begin{array}{r}8,3 \\
26,5 \\
13,0 \\
29,3 \\
21,6\end{array}$ \\
\hline \multicolumn{14}{|l|}{ Pernambuco } \\
\hline $\begin{array}{l}\text { Ouricuri } \\
\text { Triunfo } \\
\text { Surubim } \\
\text { Arcoverde } \\
\text { Cabrobó } \\
\text { Garanhuns } \\
\text { Recife } \\
\text { Petrolina }\end{array}$ & $\begin{array}{r}7,0 \\
2,4 \\
47,7 \\
6,0 \\
16,2 \\
13,8 \\
3,7 \\
20,6\end{array}$ & $\begin{array}{r}9,1 \\
4,8 \\
38,2 \\
8,7 \\
23,1 \\
15,6 \\
5,5 \\
28,8\end{array}$ & $\begin{array}{r}5,4 \\
3,6 \\
22,8 \\
5,8 \\
12,6 \\
11,9 \\
6,5 \\
20,7\end{array}$ & $\begin{array}{r}8,5 \\
7,7 \\
17,4 \\
6,5 \\
12,5 \\
14,5 \\
8,7 \\
21,4\end{array}$ & $\begin{array}{r}11,0 \\
15,4 \\
15,0 \\
9,6 \\
18,0 \\
15,9 \\
12,1 \\
34,0\end{array}$ & $\begin{array}{l}23,6 \\
29,6 \\
12,1 \\
15,0 \\
32,4 \\
19,5 \\
19,7 \\
48,4\end{array}$ & $\begin{array}{l}30,7 \\
38,2 \\
15,7 \\
15,6 \\
44,5 \\
23,6 \\
22,8 \\
55,5\end{array}$ & $\begin{array}{l}33,5 \\
28,0 \\
23,4 \\
22,6 \\
63,6 \\
24,3 \\
23,4 \\
55,5\end{array}$ & $\begin{array}{l}37,4 \\
19,4 \\
35,3 \\
23,8 \\
71,1 \\
23,7 \\
22,5 \\
61,5\end{array}$ & $\begin{array}{r}23,8 \\
8,2 \\
46,1 \\
20,0 \\
50,3 \\
15,0 \\
13,2 \\
50,0\end{array}$ & $\begin{array}{r}12,8 \\
0,8 \\
57,5 \\
7,7 \\
27,9 \\
4,4 \\
1,8 \\
33,0\end{array}$ & $\begin{array}{r}8,1 \\
1,2 \\
48,6 \\
7,2 \\
19,3 \\
7,5 \\
5,8 \\
25,1\end{array}$ & $\begin{array}{l}17,6 \\
13,3 \\
31,7 \\
12,4 \\
32,6 \\
15,8 \\
12,1 \\
37,9\end{array}$ \\
\hline \multicolumn{14}{|l|}{ Alagoas } \\
\hline $\begin{array}{l}\text { Palmeira dos Índios } \\
\text { Maceió } \\
\text { Coruripe }\end{array}$ & $\begin{array}{r}32,9 \\
2,5 \\
27,7\end{array}$ & $\begin{array}{r}19,1 \\
4,3 \\
5,7\end{array}$ & $\begin{array}{r}14,5 \\
4,0 \\
19,3\end{array}$ & $\begin{array}{l}8,1 \\
4,1 \\
6,5\end{array}$ & $\begin{array}{l}3,4 \\
6,3 \\
4,9\end{array}$ & $\begin{array}{r}3,4 \\
10,7 \\
5,2\end{array}$ & $\begin{array}{r}3,4 \\
10,3 \\
3,3\end{array}$ & $\begin{array}{r}6,3 \\
12,3 \\
8,6\end{array}$ & $\begin{array}{l}12,9 \\
12,8 \\
14,2\end{array}$ & $\begin{array}{r}32,2 \\
9,5 \\
21,3\end{array}$ & $\begin{array}{r}49,0 \\
1,0 \\
16,0\end{array}$ & $\begin{array}{r}43,4 \\
7,3 \\
27,5\end{array}$ & $\begin{array}{r}19,0 \\
7,1 \\
13,4\end{array}$ \\
\hline \multicolumn{14}{|l|}{ Sergipe } \\
\hline $\begin{array}{l}\text { Aracaju } \\
\text { Propriá }\end{array}$ & $\begin{array}{l}18,3 \\
33,4\end{array}$ & $\begin{array}{l}26,5 \\
25,8\end{array}$ & $\begin{array}{l}19,7 \\
19,3\end{array}$ & $\begin{array}{l}18,0 \\
11,9\end{array}$ & $\begin{array}{r}27,6 \\
4,8\end{array}$ & $\begin{array}{r}33,0 \\
6,9\end{array}$ & $\begin{array}{r}38,0 \\
5,7\end{array}$ & $\begin{array}{l}36,0 \\
10,6\end{array}$ & $\begin{array}{l}33,5 \\
14,9\end{array}$ & $\begin{array}{l}21,8 \\
17,4\end{array}$ & $\begin{array}{r}7,5 \\
27,2\end{array}$ & $\begin{array}{l}17,2 \\
34,3\end{array}$ & $\begin{array}{l}24,8 \\
17,7\end{array}$ \\
\hline \multicolumn{14}{|l|}{ Ceará } \\
\hline $\begin{array}{l}\text { Acaraú } \\
\text { Sobral } \\
\text { Fortaleza } \\
\text { Guaramiranga } \\
\text { Jaguaruana } \\
\text { Crateús } \\
\text { Quixeramobim } \\
\text { Morada Nove } \\
\text { Iguatu } \\
\text { Barbalha }\end{array}$ & $\begin{array}{r}51,7 \\
31,9 \\
20,3 \\
32,6 \\
19,7 \\
3,7 \\
27,5 \\
20,2 \\
1,1 \\
0,3\end{array}$ & $\begin{array}{r}33,3 \\
19,2 \\
19,9 \\
34,4 \\
15,5 \\
3,3 \\
17,3 \\
9,5 \\
2,1 \\
0,5\end{array}$ & $\begin{array}{r}25,5 \\
6,3 \\
9,0 \\
31,5 \\
7,6 \\
3,4 \\
12,7 \\
4,5 \\
1,0 \\
0,5\end{array}$ & $\begin{array}{r}19,5 \\
3,8 \\
8,4 \\
24,0 \\
3,9 \\
4,4 \\
4,8 \\
2,0 \\
3,1 \\
1,5\end{array}$ & $\begin{array}{r}22,0 \\
1,7 \\
7,0 \\
28,2 \\
3,2 \\
6,1 \\
2,7 \\
1,6 \\
9,2 \\
3,6\end{array}$ & $\begin{array}{r}29,7 \\
1,6 \\
8,4 \\
30,8 \\
4,3 \\
12,9 \\
1,8 \\
1,3 \\
15,0 \\
7,4\end{array}$ & $\begin{array}{r}42,6 \\
5,2 \\
13,1 \\
33,0 \\
5,5 \\
18,8 \\
3,8 \\
2,5 \\
22,5 \\
9,0\end{array}$ & $\begin{array}{r}86,8 \\
10,7 \\
26,5 \\
32,5 \\
12,7 \\
19,5 \\
12,5 \\
6,1 \\
16,3 \\
8,7\end{array}$ & $\begin{array}{r}121,5 \\
20,2 \\
31,2 \\
61,0 \\
21,7 \\
16,1 \\
21,9 \\
16,0 \\
9,8 \\
6,7\end{array}$ & $\begin{array}{r}138,3 \\
34,1 \\
37,8 \\
64,7 \\
31,2 \\
7,5 \\
35,3 \\
28,5 \\
1,2 \\
2,3\end{array}$ & $\begin{array}{r}125,2 \\
36,0 \\
26,2 \\
56,8 \\
32,1 \\
2,4 \\
37,4 \\
32,6 \\
0,6 \\
0,6\end{array}$ & $\begin{array}{r}75,9 \\
37,1 \\
27,5 \\
43,2 \\
24,4 \\
2,5 \\
31,0 \\
25,8 \\
0,7 \\
0,8\end{array}$ & $\begin{array}{r}64,3 \\
17,3 \\
19,6 \\
39,4 \\
15,1 \\
8,4 \\
17,4 \\
12,6 \\
6,9 \\
3,5\end{array}$ \\
\hline \multicolumn{14}{|l|}{ Piauí } \\
\hline $\begin{array}{l}\text { Parnaíba } \\
\text { Piripiri } \\
\text { Terezina } \\
\text { São Félix do Piauí } \\
\text { Floriano } \\
\text { Uruçuí } \\
\text { Picos } \\
\text { São João do Piauí } \\
\text { Paulistana } \\
\text { Bom Jesus do Piauí } \\
\text { Caracol }\end{array}$ & $\begin{array}{r}24,6 \\
2,6 \\
0,2 \\
0,7 \\
0,9 \\
4,0 \\
2,3 \\
0,2 \\
15,5 \\
1,6 \\
6,2\end{array}$ & $\begin{array}{r}22,2 \\
1,8 \\
0,2 \\
0,6 \\
1,1 \\
4,6 \\
2,9 \\
0,2 \\
23,8 \\
1,1 \\
7,4\end{array}$ & $\begin{array}{r}13,8 \\
2,0 \\
0,4 \\
0,7 \\
2,2 \\
4,1 \\
1,6 \\
0,4 \\
24,0 \\
0,9 \\
4,6\end{array}$ & $\begin{array}{r}12,2 \\
1,0 \\
0,6 \\
0,9 \\
2,7 \\
5,4 \\
4,5 \\
0,5 \\
28,7 \\
2,6 \\
3,8\end{array}$ & $\begin{array}{r}12,6 \\
0,6 \\
1,5 \\
1,3 \\
4,1 \\
6,4 \\
8,5 \\
0,3 \\
51,8 \\
3,0 \\
3,6\end{array}$ & $\begin{array}{r}14,2 \\
0,6 \\
3,8 \\
5,6 \\
5,6 \\
10,3 \\
15,9 \\
0,8 \\
78,3 \\
4,1 \\
6,9\end{array}$ & $\begin{array}{r}19,6 \\
1,1 \\
4,3 \\
4,5 \\
11,1 \\
11,2 \\
21,2 \\
0,8 \\
105,6 \\
3,7 \\
8,4\end{array}$ & $\begin{array}{r}39,3 \\
2,0 \\
4,3 \\
5,2 \\
10,5 \\
10,6 \\
17,9 \\
0,7 \\
108,1 \\
2,4 \\
12,2\end{array}$ & $\begin{array}{r}73,5 \\
3,2 \\
2,8 \\
3,7 \\
13,1 \\
10,8 \\
17,2 \\
0,3 \\
111,5 \\
2,2 \\
18,7\end{array}$ & $\begin{array}{r}73,7 \\
5,5 \\
1,7 \\
1,1 \\
4,1 \\
6,7 \\
4,7 \\
0,4 \\
75,6 \\
2,1 \\
19,3\end{array}$ & $\begin{array}{r}63,7 \\
5,2 \\
0,5 \\
0,5 \\
1,1 \\
2,2 \\
3,5 \\
0,5 \\
26,5 \\
2,3 \\
11,2\end{array}$ & $\begin{array}{r}40,1 \\
2,9 \\
0,3 \\
0,5 \\
0,9 \\
2,0 \\
4,0 \\
0,2 \\
13,7 \\
1,6 \\
6,2\end{array}$ & $\begin{array}{r}34,1 \\
2,4 \\
1,7 \\
2,1 \\
4,8 \\
6,5 \\
8,7 \\
0,4 \\
55,3 \\
2,3 \\
9,0\end{array}$ \\
\hline \multicolumn{14}{|l|}{ Maranhão } \\
\hline $\begin{array}{l}\text { Turiaçu } \\
\text { São Luiz } \\
\text { Zé Doca } \\
\text { Chapadinha } \\
\text { Bacabal } \\
\text { Caxias }\end{array}$ & $\begin{array}{r}14,8 \\
9,5 \\
5,0 \\
4,2 \\
1,3 \\
1,4\end{array}$ & $\begin{array}{r}12,6 \\
7,5 \\
6,4 \\
4,4 \\
0,9 \\
1,9\end{array}$ & $\begin{array}{l}9,0 \\
5,7 \\
4,1 \\
3,0 \\
1,2 \\
2,1\end{array}$ & $\begin{array}{l}6,5 \\
2,8 \\
1,6 \\
1,7 \\
0,4 \\
3,9\end{array}$ & $\begin{array}{l}6,2 \\
1,6 \\
1,2 \\
1,3 \\
0,3 \\
6,2\end{array}$ & $\begin{array}{r}7,7 \\
1,7 \\
0,9 \\
1,1 \\
0,1 \\
11,8\end{array}$ & $\begin{array}{r}10,7 \\
3,0 \\
1,6 \\
1,6 \\
0,2 \\
11,9\end{array}$ & $\begin{array}{r}19,7 \\
7,8 \\
3,1 \\
2,9 \\
0,9 \\
10,0\end{array}$ & $\begin{array}{r}37,6 \\
17,6 \\
8,0 \\
5,4 \\
2,2 \\
7,8\end{array}$ & $\begin{array}{r}29,8 \\
20,5 \\
11,8 \\
8,2 \\
3,4 \\
4,6\end{array}$ & $\begin{array}{r}29,9 \\
17,3 \\
13,1 \\
8,2 \\
3,5 \\
2,3\end{array}$ & $\begin{array}{r}24,7 \\
16,2 \\
10,3 \\
8,6 \\
2,1 \\
2,7\end{array}$ & $\begin{array}{r}17,4 \\
9,3 \\
5,6 \\
4,2 \\
1,4 \\
5,6\end{array}$ \\
\hline
\end{tabular}


Continuação da página anterior

\begin{tabular}{|c|c|c|c|c|c|c|c|c|c|c|c|c|c|}
\hline \multirow{2}{*}{ Localidades } & \multicolumn{12}{|c|}{ Meses } & \multirow{2}{*}{ Média } \\
\hline & Jan & $\mathrm{Fev}$ & Mar & $\mathrm{Abr}$ & Mai & Jun & Jul & Ago & Set & Out & Nov & Dez & \\
\hline \multicolumn{14}{|l|}{ Maranhão } \\
\hline Imperatriz & 0,8 & 0,9 & 1,4 & 2,5 & 3,0 & 5,0 & 6,9 & 4,6 & 2,2 & 1,0 & 1,0 & 0,9 & 2,5 \\
\hline Barra do Corda & 0,9 & 1,3 & 1,8 & 3,2 & 3,5 & 5,1 & 4,2 & 4,4 & 3,2 & 1,4 & 1,0 & 1,6 & 2,6 \\
\hline Colinas & 0,6 & 0,9 & 1,0 & 2,4 & 4,3 & 7,8 & 8,1 & 6,4 & 3,5 & 1,7 & 0,6 & 0,7 & 3,1 \\
\hline Carolina & 0,8 & 0,9 & 0,7 & 0,5 & 0,4 & 1,2 & 3,0 & 3,3 & 4,3 & 2,1 & 1,3 & 1,2 & 1,6 \\
\hline Alto Paranaíba & 0,9 & 1,1 & 0,8 & 0,4 & 0,1 & 0,0 & 0,1 & 0,4 & 1,0 & 1,2 & 1,0 & 1,2 & 0,7 \\
\hline \multicolumn{14}{|l|}{ Bahia } \\
\hline Remanso & 11,8 & 11,8 & 18,7 & 19,7 & 18,9 & 32,6 & 31,9 & 36,7 & 53,3 & 40,1 & 17,5 & 22,7 & 26,3 \\
\hline Paulo Afonso & 17,9 & 12,4 & 5,6 & 6,8 & 7,9 & 11,8 & 16,7 & 22,7 & 35,2 & 38,4 & 34,3 & 25,7 & 19,6 \\
\hline Ipibetuba & 1,7 & 2,1 & 1,5 & 2,3 & 2,4 & 3,0 & 3,7 & 4,4 & 5,3 & 3,0 & 2,1 & 2,2 & 2,8 \\
\hline Monte Santo & 4,0 & 5,4 & 3,3 & 4,4 & 4,7 & 6,0 & 6,8 & 8,3 & 12,8 & 13,0 & 9,4 & 6,5 & 7,0 \\
\hline Barra & 5,5 & 5,1 & 4,2 & 3,4 & 1,6 & 2,6 & 4,1 & 8,6 & 17,7 & 14,9 & 10,4 & 9,2 & 7,3 \\
\hline Irecê & 8,6 & 10,6 & 8,4 & 11,9 & 19,1 & 30,8 & 29,5 & 25,4 & 18,3 & 18,8 & 10,5 & 8,6 & 16,7 \\
\hline Jacobina & 24,9 & 31,2 & 24,5 & 18,8 & 21,8 & 25,9 & 32,5 & 40,8 & 52,7 & 54,5 & 31,7 & 17,1 & 31,4 \\
\hline Serrinha & 12,7 & 8,6 & 6,5 & 5,5 & 3,3 & 1,7 & 2,9 & 6,3 & 12,6 & 24,6 & 26,1 & 21,5 & 11,0 \\
\hline Salvador & 4,9 & 5,1 & 4,3 & 3,2 & 3,2 & 2,1 & 2,5 & 3,6 & 3,8 & 5,3 & 6,8 & 5,2 & 4,2 \\
\hline Barreiras & 3,4 & 2,7 & 2,4 & 1,2 & 0,8 & 0,5 & 0,7 & 1,3 & 2,2 & 1,9 & 2,4 & 3,6 & 1,9 \\
\hline Lençóis & 0,9 & 1,3 & 0,9 & 1,3 & 1,1 & 1,7 & 0,9 & 1,4 & 1,2 & 1,4 & 1,1 & 0,7 & 1,2 \\
\hline Itaberaba & 3,0 & 3,9 & 2,1 & 1,8 & 2,4 & 2,5 & 3,5 & 5,9 & 9,8 & 12,3 & 10,2 & 9,1 & 5,5 \\
\hline Alagoinhas & 4,5 & 8,0 & 4,2 & 4,4 & 4,6 & 5,3 & 5,4 & 6,2 & 7,7 & 7,6 & 4,4 & 6,0 & 5,7 \\
\hline Correntina & 0,3 & 0,6 & 0,2 & 1,1 & 1,9 & 5,5 & 5,3 & 5,2 & 3,8 & 3,0 & 1,1 & 0,4 & 2,4 \\
\hline Bom Jesus da Lapa & 0,9 & 2,4 & 1,2 & 3,0 & 3,1 & 6,5 & 4,5 & 4,2 & 3,1 & 3,8 & 2,2 & 1,4 & 3,0 \\
\hline Jaguaquara & 2,7 & 2,9 & 2,1 & 1,5 & 0,9 & 0,8 & 1,5 & 2,0 & 3,6 & 4,8 & 4,7 & 5,5 & 2,7 \\
\hline Caetité & 9,6 & 22,9 & 20,6 & 35,1 & 53,1 & 52,1 & 51,2 & 64,4 & 76,6 & 60,3 & 29,9 & 12,6 & 40,7 \\
\hline Vitória da Conquista & 4,0 & 8,9 & 5,5 & 3,8 & 5,7 & 5,4 & 6,1 & 7,0 & 11,4 & 10,0 & 6,0 & 6,4 & 6,7 \\
\hline Ilhéus & 2,2 & 4,0 & 3,8 & 19,8 & 13,9 & 25,5 & 16,9 & 12,8 & 12,7 & 8,1 & 11,0 & 3,5 & 11,2 \\
\hline Carinhana & 1,7 & 5,7 & 3,9 & 4,2 & 3,3 & 4,3 & 5,0 & 10,4 & 11,6 & 8,1 & 3,1 & 2,1 & 5,3 \\
\hline Guaratinga & 2,9 & 1,0 & 1,2 & 0,8 & 0,6 & 0,2 & 0,6 & 1,0 & 1,5 & 2,6 & 3,5 & 3,0 & 1,6 \\
\hline Caravelas & 12,6 & 6,4 & 7,4 & 4,2 & 1,8 & 0,8 & 2,4 & 5,5 & 8,8 & 11,0 & 15,3 & 13,6 & 7,5 \\
\hline
\end{tabular}

No Estado da Bahia, a DPMA alcançou o seu máximo em Remanso, com 26,288 $\mathrm{W} \mathrm{m}^{-2}$. Por sua vez, a localidade de menor DPMA foi Lencóis, com apenas $1,157 \mathrm{~W} \mathrm{~m}^{-2}$. Tchinda et al. (2000) constataram que no norte de Camarões a DPMA foi, em geral, igual ou superior a $15 \mathrm{~W} \mathrm{~m}^{-2}$, sendo que em outubro ela foi de 11,4 $\mathrm{W} \mathrm{m}^{-2} \mathrm{e}$, em março, alcançou 29,27 $\mathrm{W} \mathrm{m}^{-2}$. Aqueles pesquisadores concluíram que a energia solar disponível naquele país era bem maior que a energia eólica.

Dentre os Estados estudados, aquele que apresentou a menor potência eólica foi o Estado do Maranhão. O Estado do Rio Grande do Norte, entretanto, foi o que registrou a maior potência eólica. As localidades que apresentaram valores mensais de densidade da potência eólica média (DPEM) superiores a $100 \mathrm{~W} \mathrm{~m}^{-2}$, foram Paulistana, PI (julho, agosto e setembro) e Acaraú, CE (setembro, outubro e novembro). Nesta última localidade, a DPMM alcançou, no mês de outubro, a $138,302 \mathrm{~W} \mathrm{~m}^{-2}$. Nfaoui et al. (1998) observaram que em Taza, Marrocos, a DPMA foi de $900 \mathrm{~W} \mathrm{~m}^{-2}$, para o nível de $10 \mathrm{~m}$, e que o mesmo aumentou em $85 \%$ quando se considerou a sua projeção a $30 \mathrm{~m}$; já na Argélia, a DPMA variou de $37 \mathrm{~W} \mathrm{~m}^{-2}$, em Skikada, a $161 \mathrm{Wm}^{-2}$, em Tiaret (Merzouk, 2000).

A causa da variabilidade espacial do potencial eólico obtido pode estar relacionada a diversos fatores, dentre eles a eventual localização das estações com relação à topografia local, e efeitos micrometeorológicos e de mesoescala, que não são detectáveis a partir de dados em escala sinótica (convecção, efeitos de vale-montanha, etc.). As potências máximas ocorrem entre junho e dezembro, e as mínimas entre março e maio, à exceção do sul da Bahia, que apresenta máxima entre junho e setembro. A razão climatológica desse comportamento diferenciado em relação ao restante do Nordeste, deve-se ao fato da influência de sistemas sinóticos de latitudes médias provenientes do sul do Brasil, que atuam na época do inverno. Para o litoral, os ventos alísios favorecem a penetração da brisa marítima no continente durante os períodos de máxima. Nessas épocas, as maiores intensidades de potência eólica encontram-se no interior do continente (Bandeira, 1990). As demais razões da variabilidade da potência eólica do Nordeste, provocada por influências meteorológicas, não são abordadas e aprofundadas neste trabalho nem, também, o rendimento esperado por aerogeradores, em função dessa potência encontrada.

\section{CONCLUSÕES}

1. A direção predominante do vento no Nordeste do Brasil é de Leste e que a distribuição de Weibull representou adequadamente a distribuição de freqüências da velocidade do vento.

2. A potência eólica no Nordeste do Brasil evoluiu de zonas de baixas potencialidades, como no centro-oeste, com valores da ordem de $5 \mathrm{~W} \mathrm{~m}^{-2}$, até zonas de máximas potencialidades, como no litoral norte, com valores da ordem de $65 \mathrm{~W} \mathrm{~m}^{-2}$.

\section{AGRADECIMENTOS}

Ao Conselho Nacional de Desenvolvimento Científico e Tecnológico, CNPq, pela bolsa de Produtividade em Pesquisa, 
concedida ao primeiro autor; ao Departamento de Ciências Atmosféricas da UFCG, e ao Instituto Nacional de Meteorologia, pelos dados usados na pesquisa.

\section{LITERATURA CITADA}

Abramowitz, M; Stegun, I.A. Handbook of mathematical functions, with formulas, graphs and mathematical tables, Washington: Dover Publications Incorporated, 1972. 1046p. Bandeira, M.M. Regionalização do regime eólico no Nordeste do Brasil. Campina Grande: Departamento de Ciências Atmosféricas, DCA/UFPB. 1990. 62p. Dissertação Mestrado

Bastos, E.J.B.; Ceballos, J.C; Sobral, Z.R. Zoneamento eólico do Nordeste. Campina Grande, 1986, 70p. Relatório Técnico CHESF/UFPB

Borhan, Y. Mesoscale interactions on wind energy potential in the northern Aegean region: A case study. Renewable \& Sustainable Energy Reviews, Amsterdam, v.2, p.353-360, 1998.

Galvani, E.; Klosowski, E.S.; Cunha, A.R. da. Caracterização da direção predominante do vento em Maringá, PR. Revista Brasileira de Agrometeorologia, Santa Maria, v.7, n.1, p.81-90, 1999.

Garcia, A.; Torres, J.L.; Prieto, E.; De Francisco, A. Fitting wind speed distributions: A case study. Solar Energy, Freiburg, v.62, n.2, p.139-144, 1998.

Justus, C.G; Mikail, A. Height variation of wind speed and wind distribution. Geophysical Research Letters, Boston, v.3, p.261-264, 1976.
Mayhoub, A.B; Azzam, A. A survey on the assessment of wind energy potential in Egypt. Renewable Energy, Oxford, v.11, n.2, p.235-247, 1997.

Merzouk, N.K. Wind energy potential of Algeria. Renewable Energy, Oxford, v.21, p.553-562, 2000.

Nfaoui, H; Buret, J; Sayigh, A.A.M. Wind characteristics and wind energy potential in Marrocos. Solar Energy, Freiburg, v.63, n.1, p.51-60, 1998.

Sahin, A.Z; Aksakal, A. Wind power energy potential at the northeastern region of Saudi Arabia. Renewable Energy, Oxford, v.14, p.435-440, 1998.

Silva, B.B.da; Alves, J.J.A; Azevedo, F.G.B.de. Potencial eólico do Estado da Paraíba. Congresso Brasileiro de Agrometeorologia, 12, Reunião Latino-americana de Agrometeorologia, 3 , 2001, Fortaleza. Anais... Fortaleza: FUNCEME, 2001, v.2, p.577-578.

Sopian, K; Othman, M.Y.H; Wirsat, A. The wind energy potential of Malaysia. Renewable Energy, Oxford, v.6, p.1005-1016, 1995.

Souza, A; Granja, S.C. Estimativa dos parâmetros "C" e "K" do modelo de Weibull e da direção dos ventos para Campo Grande e Dourados, MS, Brasil. Revista Brasileira de Agrometeorologia, Santa Maria, v.5, n.1, p.109-114, 1997.

Tchinda, R; Kenjio, J; Kaptouom, E; Njomo, D. Estimation of mean wind energy availabe in far north Cameroon. Energy Conversion \& Management, Amsterdam, v.41, p.1917-1929, 2000.

Tolun, S; Ments, S; Aslan, Z; Wukselen, M.A. The wind energy potential of Gokçeada in the northern Aegean sea. Renewable Energy, Oxford, v.6, p.679-685, 1995. 